\title{
Cardiologists and the management of blood sugar: a Chinese perspective
}

\author{
Da-Yi Hu and Yi-Fang Guo
}

In past decades, the diagnosis and management of diabetes mellitus has largely been viewed as the responsibility of diabetes experts. Over recent years, however, evidence has accumulated to indicate that the prevalence of diabetes and the prediabetic state is remarkably high in patients with cardiovascular disease.

The results of the 2004 Euro Heart Survey shook the cardiology community. This study showed that abnormal glucose metabolism was far more common than normal glucose regulation in patients with coronary artery disease (CAD). Shortly after the publication of this research, the China Heart Survey - the only large-scale study on a Chinese population in this field to date- reported that many patients with CAD also had previously undiagnosed diabetes and impaired glucose tolerance (IGT), and that the prevalence of IGT was much higher in patients with CAD than in those without CAD. These findings shocked the Chinese cardiology community, prompting many to debate whether cardiologists should, in fact, be responsible for screening of carbohydrate metabolism among patients with cardiovascular disease.

In terms of the number of individuals with diabetes, China ranks second in the world with at least 20 million cases. Indeed, in China an increasing number of physicians are realizing that screening for diabetes and prediabetes in their patients with CAD could be important in the early risk assessment of individuals in this high-risk population, who will most probably require intensive intervention. After all, initial studies have shown that the management of postprandial glucose and cholesterol has considerable benefits in these individuals.

According to results from Chinese epidemiological studies and trials from other areas of the globe, individuals with type 2 diabetes have a two to four times greater risk of CAD and stroke than the general population, and approximately $80 \%$ of all diabetes-related deaths are associated with cardiovascular diseases. On the basis of these findings, diabetes has been believed

\section{In terms of the number of individuals with diabetes, China ranks second in the world with at least 20 million} cases.

D-YHu is Director of the Heart Center at People's Hospital of Peking University, Beijing, and Y-F Guo is Head of the Cardiology Division at the Geriatric Institute, Hebei Provincial People's Hospital, Shijiazhuang, China.

\section{Competing interests}

The author declared no competing interests.

www.nature.com/clinicalpractice doi:10.1038/ncpcardio1325 to be an independent risk factor for atherosclerosis, just as important as hypertension, dyslipidemia and smoking. Similarly, studies have shown that IGT (or postprandial hyperglycemia) is also associated with a substantially increased risk of CAD. So far, however, little evidence has been obtained about whether patients with IGT benefit from intervention with hypoglycemic agents in terms of cardiovascular end points. Although some studies have shown that patients with diabetes might benefit, findings have been mixed. Recent years have seen the publication of a series of milestone trials analyzing glycemic control (ACCORD, ADVANCE and VADT), which found a nonsignificant relationship between blood sugar reduction and decreased incidence of cardiovascular events in diabetic patients at high cardiovascular risk. We have to conclude, therefore, that we should treat patients with abnormal glucose metabolism at the early stage of diabetes if we are to attain a significant reduction in the risk of cardiovascular events.

To clarify whether patients with IGT would benefit from intervention with hypoglycemic agents in terms of cardiovascular end points, the Acarbose Cardiovascular Evaluation (ACE) trial has been developed. This trial will prospectively recruit 7,500 individuals with CAD but without diabetes who have IGT. Patients will receive either acarbose or placebo for at least 4 years. The primary end point is the occurrence of a first cardiovascular event, with the development of new-onset diabetes as a secondary end point. Final trial results are expected in 2012. Without a doubt, the conclusions of the ACE trial will expand our knowledge of the primary prevention of diabetes and of the secondary prevention of CAD by managing prediabetes.

The problem of diabetes is acute and we need findings from studies such as ACE to tackle this growing issue effectively. Importantly, Chinese cardiologists are now beginning to pay attention to hyperglycemia in their patients with cardiovascular disease. 